\title{
Management Of Mandibular Lateral Incisor Agenesis With Skeletal Class III Malocclusion by Space Closing Technique
}

\author{
Fidiyah Inayati, I Gusti Aju Wahju Ardani \\ Department of Orthodontics, Faculty of Dental Medicine \\ Universitas Airlangga \\ Surabaya - Indonesia
}

\begin{abstract}
Background: Patients with congenital absence of a mandibular lateral incisor are often found having difficulty in achieving adequate functional occlusion. It may affect esthetics, mastication, speech, and occlusal balance. Purpose: This paper reported an agenesis treatment of one mandibular lateral incisor case using a space closure method. Case: A twenty-three years old female patient with agenesis of tooth 42, mandibular anterior crowding, multiple diastema on mandibular anterior teeth, and skeletal class III malocclusion. Case Management: Space closure method was chosen to correct the agenesis by considering the class III skeletal malocclusion and multiple diastema condition. Conclusion: Space closure method treatment improved the patient's facial and dental esthetics, and it provided a good functional occlusion, despite the absence of a mandibular lateral incisor, which generally impairs the adequate incisal guidance.
\end{abstract}

Keywords: agenesis, space closing method, skeletal class III malocclusion, multiple diastema.

Correspondence: I Gusti Aju Wahju Ardani, Department of Orthodontics, Faculty of Dental Medicine, Universitas Airlangga Jl. Prof. Dr. Moestopo No. 47, Surabaya 60132 - Indonesia, Phone: (031)5030255 Email: wahju_ardani@fkg. unair.ac.id

\section{INTRODUCTION}

Agenesis means the absence of development of one or more elements of a permanent tooth due to the absence of formation or non-grown permanent tooth bud. Dental agenesis is classified according to the number of teeth that are not formed, excluding the third molars. ${ }^{1}$ Hypodontia is a term used to denote agenesis of one to five teeth; oligodontia, when six or more teeth are lost congenitally; and anodontia refers to the absence of complete tooth formation. Agenesis of one or more tooth elements is the most common dental development anomaly in humans. ${ }^{1,2}$ Among various populations, the prevalence of hypodontia varies from $4.19 \%$ in India to $11.3 \%$ in Ireland. ${ }^{2}$ The agenesis prevalence of the European populations is estimated to be $0.08 \%$. Research suggests that females have higher agenesis prevalence than males. ${ }^{3}$ The most common agenesis of permanent teeth, excluding third molars, are mandibular second premolars (11.3\%) followed by mandibular incisors $(6.9 \%)$ and teeth lateral maxillary incisors $(6.5 \%)$. Most studies have shown congenital agenesis to occur in one tooth, showing unilateral, not bilateral events. However, there have been a number of reports of hypodontia, which shows bilateral tooth loss. As far as bilateral agenesis is concerned, central mandibular incisors are common., ${ }^{2,4}$ Etiologic agenesis may be related to nutritional, traumatic, infectious, genetic, or phylogenetic factors. ${ }^{5}$ Most dental agenesis cases are genetic, caused by chromosomal defects or mutations that occur during DNA chromosome replication. A large number of non-Hox genes are involved in this phenomenon (MSX141, PAX98, WNT10A32, etc.) ${ }^{6,7}$

It is difficult to achieve adequate functional occlusion in patients with lateral maxillary incisor agenesis, especially when the patient has excessive overbite or overjet and Bolton tooth discrepancy with excess in the mandibular anterior teeth. In such a condition, the anterior and lateral guidance will be disturbed. ${ }^{5}$ For non-adult patients, treatment option includes space maintenance with or without permanent dentures, followed by a fixed implant in adult age. For adult patients, orthodontic treatments such as implant placement or sealing the remaining space, completed with three mandibular incisors, are the viable options. This paper reports the treatment of agenesis of one mandibular lateral incisor case using the space closure method. 


\section{CASE}

A 23-year-old female patient visited Universitas Airlangga Dental Hospital with crowding upper teeth and multiple diastema on lower teeth. The lower jaw was more prominent than the upper jaw. The patient's medical history showed no disease that may affect the growth and development of teeth and oral mouth. The oral hygiene of the patient was good. There was no cavity found. Family history confirmed that her father and mother did not have dental abnormalities. The general examination found that the patient was in good condition. An extraoral examination showed a concave face profile and normal lips muscle tone (Figure 1). During an intraoral examination, crowded maxillary anterior teeth, multiple diastema on mandibular anterior teeth, and absence of the right lateral incisor (Figure 2) were found. The overjet was minus $3 \mathrm{~mm}$, and the overbite was $1 \mathrm{~mm}$. The first molar relation was Angle Class III. The right canine relationship was class III, and the left canine relationship was Angle class I. There was a $1 \mathrm{~mm}$ shift of the median line of the maxilla to the right, and the lower jaw was $2 \mathrm{~mm}$ to the right. The model discrepancy, the amount of space lacking for the maxilla, was $3 \mathrm{~mm}$, whereas for the lower jaw the amount of excess space was $3 \mathrm{~mm}$.

The panoramic photographs showed 28 impacted teeth, tooth 38 was extracted, and tooth 42 was agenesis (Figure

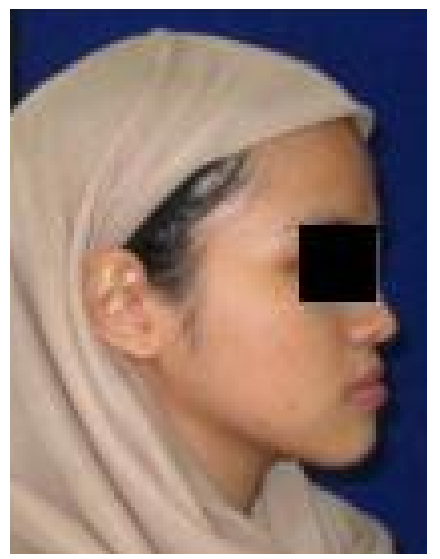

Figure 1. a concave facial profile of the patient
3). The results of cephalometry analysis showed class III skeletal malocclusion (ANB $=-1$ ), normal maxilla (SNA $=82$ ), mandibular prognosis $(\mathrm{SNB}=83)$, facial convex angle (NA-Apog $=-3$ ) showed concave profile, mandibular length $=111 \mathrm{~mm}$. In many cases, mandibular agenesis usually results in a shortening of the mandibular arch due to the absence of one tooth in the arch. ${ }^{1,5,13}$ But, in this case, there was prognathic mandibular arch, resulting in reversed bite overlap and multiple diastema. The maxillary incisor inclination angle was normal (U1-NA = 32), and the mandibular incisor inclination angle was normal (L1 - NB = 26). In Ricket's soft tissue analysis, the E line showed the normal upper lip while the lower lip showed proclination (the upper lip is $4 \mathrm{~mm}$ behind the $\mathrm{E}$ line and the lower lip is exactly on the E line). The examinations concluded that the patient's diagnosis was Class III Angle malocclusion accompanied by maxillary crowding and mandibular multiple diastema, anterior and left lateral crossbite, and shift of maxillary and mandibular median lines to the right.

\section{CASE MANAGEMENT}

The treatment began by installing fixed orthodontic appliances (MBT slot bracket 0.022) for tooth 43 using bracket 42 and bracket 43 for tooth 44 . This was to replace tooth 42 with 43 and tooth 43 with 44 considering that the patient's tooth 43 had similar shape and size to tooth 42 . The treatment was then followed with leveling and alignment

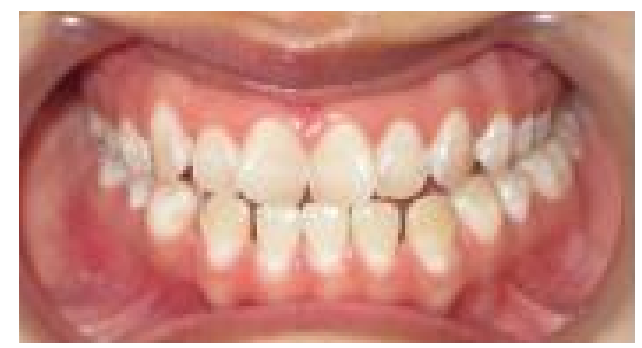

Figure 2. multiple diastema with agenesis of lateral mandibular incisor

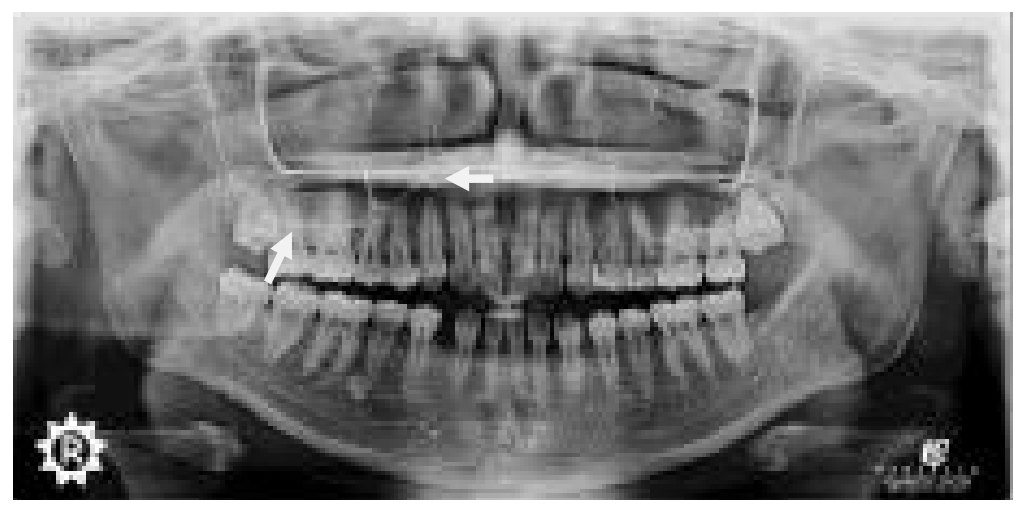

Figure 3. Panoramic radiograph pre-treatment, \#38 was extracted, \#42 agenesis. 
stages using NiTi Round 0.012 to 0.016 -inch wire, NiTi Rectangular diameter $0.016 \times 0.016$ inch and $0.016 \times 0.022$ inch, and continued with rectangular SS wire up to 0.016 $\mathrm{x} 0.022$ inch. After leveling and aligning for 8 months, and maxillary teeth crowded was corrected, lateral expansion of the left maxillary was performed to correct its lateral cross bite by using a 0.014-inch diameter of Australian wire.

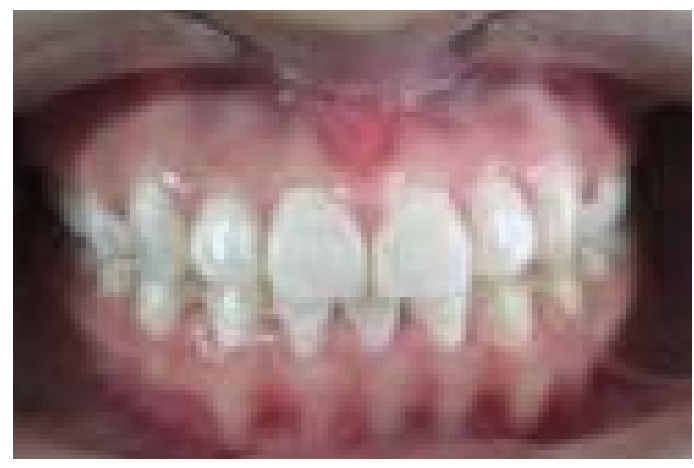

Figure 4. Intra oral post-treatment photograph, anterior cross bite and multiple diastema were corrected, dental interdigitation was better
This was done by giving an excess stop distance to the \#24 mesial teeth and distal \#27 until the left maxillary posterior bite was corrected. The next treatment was the correction of the median line shift in the maxilla and mandibula. The mandibular space closure was performed by retracting the three incisors and \#42 substitute teeth using the loop arch mechanism method with Tloop using 0.016 x 0.022 SS

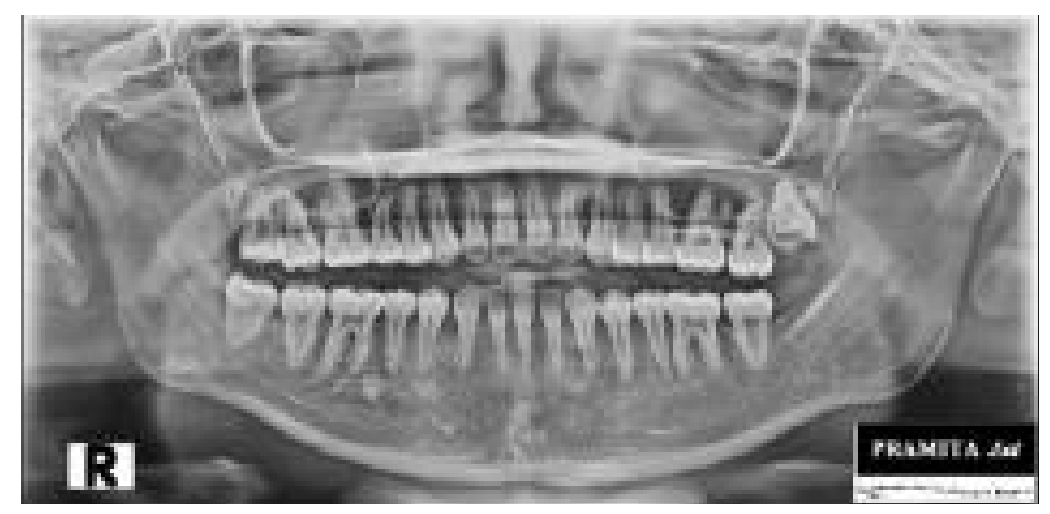

Figure 6. Panoramic radiograph post treatment showed the root parallel to the line.

Table 1. Cephalometric Analysis of Pre and Post Treatment

\begin{tabular}{|c|c|c|c|}
\hline Cephalometry & Normal & Pre Treatment & Post Treatment \\
\hline \multicolumn{4}{|c|}{ Skeletal } \\
\hline $\mathrm{SNA}^{\circ}$ & 82 & 82 & 82 \\
\hline $\mathrm{SNB}^{\circ}$ & 80 & 83 & 81 \\
\hline $\mathrm{ANB}^{\circ}$ & 2 & -1 & 1 \\
\hline Wits (mm) & 0 & -3 & -1 \\
\hline Y Axiso & 65 & 64 & 65 \\
\hline GoGn-SN。 & 32 & 35 & 38 \\
\hline $\mathrm{MP}-\mathrm{FH}^{\circ}$ & 21,9 & 28 & 29 \\
\hline \multicolumn{4}{|c|}{ Dental } \\
\hline U1-NA॰ & 26 & 32 & 33 \\
\hline U1-NA (mm) & 6,3 & 8 & 9 \\
\hline L1-NB。 & 29 & 26 & 20 \\
\hline L1-NB (mm) & 4 & 5 & 3 \\
\hline IMPA & 90 & 89 & 86 \\
\hline \multicolumn{4}{|c|}{ Soft Tissue } \\
\hline Nasolabial Angle ${ }^{\circ}$ & $110-120$ & 91 & 90 \\
\hline Upper Lip-E Line (mm) & 2-3 mm behind E Line & $4 \mathrm{~mm}$ behind $\mathrm{E}$ Line & $2 \mathrm{~mm}$ behind $\mathrm{E}$ Line \\
\hline Lower Lip-E Line (mm) & 1-2 $\mathrm{mm}$ behind $\mathrm{E}$ Line & At the $\mathrm{E}$ Line & $1 \mathrm{~mm}$ behind $\mathrm{E}$ Line \\
\hline
\end{tabular}




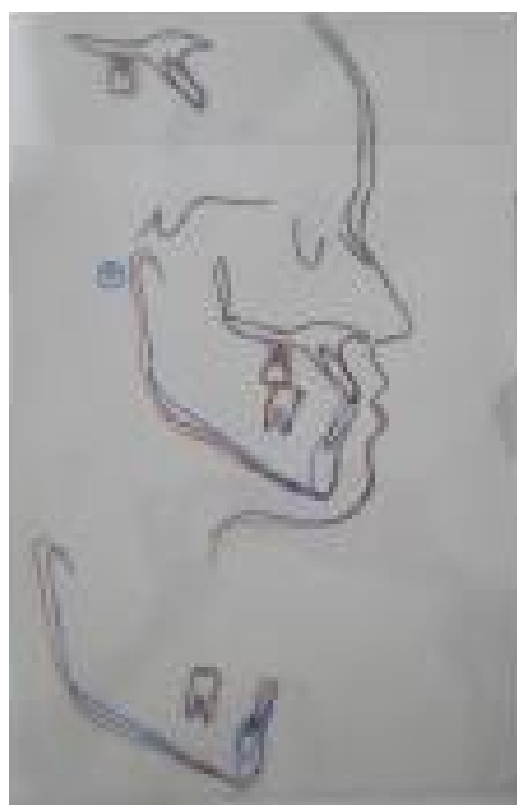

Figure 7. Superimpose tracing

wire. This was selected as class III elastic was used. This was done by considering that the anterior lower face height was normal. Thus, should there be an effect of increasing facial height, it was still within the acceptable value. The elastic was used to correct the crossbite, and at the same time, to achieve class I molar relation. Once the anterior crossbite corrected, it was followed with achieved contact and interdigitation and detailing. Finally, the finishing stage of maxillary and mandibular was done by using $0.017 \mathrm{x}$ $0.025 \mathrm{SS}$ wire.

During the final stage of treatment, Angle's class I canine relationship on the left side, Angle's class II canine relationship on the right side, and Angle's class I of both first molar were found (Figure 4). Although in the right canine relationship was categorized as class II, this was acceptable because the dental interdigitation was good, including interdigitation of substitution tooth 42 and 43 . The overjet and overbite were normal, crowding of maxillary teeth, and multiple diastema of mandibular teeth were corrected. The median line shift of the maxillary teeth was corrected. The anterior crossbite was well-corrected that the patient achieved a better face profile (Figure 5). The relationship of the left posterior teeth became normal.

The treatment result was stable due to good interdigitation, no space, normal arch shape, and the roots of teeth parallel to the line (Figure 6). The improvement satisfied the patient as reverse bite was corrected, skeletal relation became class I $(\mathrm{ANB}=1$, wits $=-1)$, point $\mathrm{B}$ retreated so that the $\mathrm{SNB}$ value decreased $(\mathrm{SNB}=83)$, the increased lower anterior facial height under normal limit (Y-Axis $=65$, GoGn-SN $=38$, $\mathrm{MP}-\mathrm{FH}=27)$. The inclination of mandibular incisors was more retrusive $(\mathrm{L} 1-\mathrm{NB}=20)$. Maxillary incisor inclination was more protrusive, but still within the normal range (U1$\mathrm{NA}=35)$ (Table 1). Superimpose tracing of cephalometric radiograph showed the changes of facial soft tissues. The upper and lower lips were straighter than the pre-treatment. The jaw was more retruded. Overall, the treatment has made the patient achieve a straighter facial profile (Figure 7).

\section{DISCUSSION}

There are two treatment options to replace agenesis, which are space opening for prostheses and space closing with nearby teeth. ${ }^{8,9,10}$ The space closing or space opening treatment shall be decided upon considerations of a number of aspects in which the ultimate goal is to achieve good aesthetics, periodontal health, and long-term function. many things must be considered to choose the treatment, including functional analysis, model analysis, and cephalometric analysis. ${ }^{11}$

In this case, mandible space closure treatment was chosen by considering the normal shape of the jaw and multiple diastema with skeletal class III malocclusion. This was consistent with the literature on indications of a selection of space closing in mandibular incisor agenesis. The indications were Angle's class I malocclusion marked by mandibular jaw or protrusion and missing incisors, missing one mandibular incisor, Angle's class III malocclusion, crowding mandibular posterior teeth, and Bolton's anterior discrepancy ratio. ${ }^{12}$

The treatment plan considerations for incisor agenesis included: skeletal profile, especially in the case of highmandibular plane angles; mechanotherapy, which tended to increase the vertical dimension must be avoided; arch length deficiency, which needed to be considered in the case of dental agenesis accompanied by smaller tooth size that can cause multiple diastema; dentialveolar protrusion; occlusion, should the relationship of the posterior segment be good, it would be maintained unless interdigitation of the posterior segment cusp to cusp. Other factors to consider were dental esthetics and dental inclination. In addition, the morphology; tooth color and mesiodistal inclination of teeth adjacent to the anterior diastema are very important to consider when the canines are considered to replace the lower lateral incisors. The canine teeth were substituted by the first premolar teeth so as to obtain satisfactory aesthetically pleasing treatment results and good stable results. The interdigitation obtained was very good, and the patient has a large mandibular arch shape; thus, it was very beneficial in this case.

The advantage of space closing agenesis treatment was the permanent biological compatibility of the teeth. The patients have their natural teeth and no prosthesis needed. The contours of the interdental gingival papilla around the natural teeth were better, teeth and gingival margins looked natural, occlusion function was adequate, and risk of mandibular temporo disorder (TMD) was reduced. A careful and meticulous orthodontic treatment combined with modern dental aesthetic materials and dental restorations using ceramic bonding, composite resins, and tooth bleaching, orthodontic space closing is the right choice. $^{11,13}$ 
In this case, the space closing treatment has improved the patient's facial and dental esthetics as well as provided a good functional occlusion. Appropriate indications and considerations, profile evaluation, the occlusion state, and the available space should be done during the treatment.

\section{REFERENCES}

1. Redua, RB and Redua PCB.Hypodontia of mandibular incisors: considerations on the orthodontic treatment.2018; Dental Press J Orthod;23(4):79-87.

2. Kagitha PK, Namineni S, Tupalli AR, Challa SK. Agenesis of Permanent Mandibular Central Incisors: A Concordant Condition in Siblings. 2016; Int J Clin Pediatr Dent;9(1):7477.

3. Porumb A, Romanul II, Dalai C, Ciavoi G, Tig IA. A Rare Case of Bilateral Agenesis of Central Lower Incisors Associated With Upper Impacted Canine- A Case Report.2015;Iran J Public Health; 45(3); 381-386.

4. Poulet $\mathrm{H}$, Poulet $\mathrm{C}$, Poulet $\mathrm{C}$. Incisor agenesis: paradigm shift A study of an orthodontic population Clinical cases.2014; J Dentofacial Anom Orthod; 17:405

5. Barros SEC, Janson G, Torres FC, Freitas MRD, Almeida RRD. Class I malocclusion treatment: Influence of a missing mandibular incisor on anterior guidance.2010; Am J Orthod Dentofacial Orthop;138:109-17.

6. Watted N, Borbely P, Watted A, Nidal G, Azzaldeen AG, Hussein MA. Multidisciplinary approach in the rehabilitation of congenitally missing lateral incisors: a new trend in daily practice.2016; Oral Health Care;1(1)1-8.

7. Dallel I, Marwen W, Abdallah SB, Tobji S, Amor AB, Canal P. Agenesis of the upper lateral incisors: Study of an orthodontic population and clinicallllustration.2018; International Orthodontics; X:1-24.

8. Hussein MA, Watted N, Abdulgani A, Borbely P. Agenesis of the upper lateral incisors: Study of an orthodontic population and clinical illustration.2015; International Journal of Maxillofacial Research;1(2):179-190.

9. Hussein MA, Watted N, Hegedus V, Peter Congenitally Missing Upper Laterals. Clinical Considerations: Orthodontic Space Closure.2015; J Dent Oral Health;1(3):014.

10. Mai A, Azzaldeen A, Nezar W, Muhammad AH. Two Treatment Approaches for Missing Maxillary Lateral Incisors: A Case.2016; IQSR-JDMS;15(7):75-85.

11. Almeida RR, Morandini ACF, Almaeda-Pedrin RR, Almeida MR, Castro RCFR, Insabralde NM. A multidisciplinary treatment of congenitally missing maxillary lateral incisors: a 14-year follow-up case report.2014; J Appl Oral Sci;22(5):465-71.

12. Ministry of Health Malaysia. Orthodontic Management Of Developmentally Missing Incisors. 2012; Clinical Practice Guidelines.

13. Aminudin MN and Sjafei A. Management Class II Division 1 Malocclusion With Congenitally Missing Mandibulary Central Incisor.2017; Proceeding Indonesian Association Of Orthodontists (IAO).2017:68-72. 